\title{
Protée
}

\section{Figures et usages du malentendu}

\section{Dominique Garand}

Volume 37, numéro 1, printemps 2009

Corps photographiques / Corps politiques

URI : https://id.erudit.org/iderudit/001560ar

DOI : https://doi.org/10.7202/001560ar

Aller au sommaire du numéro

\section{Éditeur(s)}

Département des arts et lettres - Université du Québec à Chicoutimi

\section{ISSN}

0300-3523 (imprimé)

1708-2307 (numérique)

Découvrir la revue

\section{Citer cet article}

Garand, D. (2009). Figures et usages du malentendu. Protée, 37(1), 87-101. https://doi.org/10.7202/001560ar

\section{Résumé de l'article}

Ce texte propose un exposé systématique des différents types de malentendus, des plus banals et bénins aux plus retors et pernicieux. L'objectif principal est de comprendre dans quelle mesure le malentendu peut devenir facteur de mésentente. Si l'examen rationnel des sources et des motifs de malentendus s'avère un moyen légitime de contrer leurs effets néfastes dans l'aire de la communication, il convient également de ne pas succomber à l'idéalisme et d'envisager le caractère incontournable et parfois insoluble du malentendu. Dans cette perspective, notre réflexion remet en question trois postulats de l'éthique de la communication défendue par Jürgen Habermas : la place donnée à la raison spéculative et l'efficacité d'un métadiscours explicatif dans l'élucidation des malentendus ou dans la résolution des conflits; la possibilité d'un dialogue authentique dans certaines situations de conflit ; le consensus comme visée ultime du dialogue. Alors qu'Habermas situe tout échange authentique dans l'orbite d'une prétention à la validité, nous soutenons qu'il existe plus fondamentalement chez les sujets parlants une prétention à la jouissance et que les malentendus les plus coriaces prennent racine dans ce lieu des affects. Nous soutenons par ailleurs qu'il peut exister un usage éthique du malentendu.
Ce document est protégé par la loi sur le droit d'auteur. L'utilisation des services d’Érudit (y compris la reproduction) est assujettie à sa politique d'utilisation que vous pouvez consulter en ligne.

https://apropos.erudit.org/fr/usagers/politique-dutilisation/ 


\section{HORS DOSSIER}




\title{
FIGURES ET USAGES DU MALENTENDU
}

\author{
DOMINIQUE GARAND
}

Le malentendu nous apparaît spontanément comme une source de conflit. À un point tel que «bien s'entendre» est I'expression qui désigne entre les individus un état de complicité et d'harmonie, l'exact opposé de la confrontation. Pourtant, le français introduit une nuance entre le malentendu (misunderstanding) et la mésentente (disagreement), deux mots voisins dans leur étymologie. Le premier terme, avec son accord au passé (quelque chose a eu lieu sur quoi il faut revenir), renvoie à un phénomène acoustique ou interprétatif, alors que le second désigne la complication présente d'un rapport entre individus. $C^{\prime}$ est donc à ce deuxième terme qu'il faut associer plus étroitement la conflictualité.

À la limite, il nous serait possible d'imaginer des malentendus « heureux», quand, par exemple, le fait d'avoir mal compris quelque chose nous pousse à une action dont le résultat est positif. Ces cas sont rares, sauf dans les comédies de Marivaux qui partent du principe que l'être humain, de toute façon, se ment à lui-même et aux autres, ce qui donne au malentendu (ou à la « méprise », autre terme associé) la fonction de lui révéler, à son corps défendant, son vrai désir. On connaît aussi ce célèbre aphorisme de Baudelaire:

Le monde ne marche que par le malentendu.

C'est par le malentendu universel que tout le monde s'accorde.

Car si par malheur on se comprenait, on ne pourrait jamais s'ac-

corder. (Mon cœur mis à nu, fragment 57)

Vladimir Jankélévitch, qui s'en inspire, en rajoute:

Ce n'est pas la moindre ironie de la condition humaine qu'il puisse y avoir un bon usage de cette mésintelligence si bien entendue! Les hommes sont faibles et méchants, mesquins et misérables au point que cette duperie vaut peut-être mieux pour leur misère : s'ils doivent s'accorder entre eux, puissent-ils ne pas trop se comprendre les uns les autres... Puissent-ils ne pas se comprendre trop tôt! Puissent-ils se mécomprendre!Y aurait-il par hasard plus à redouter qu'à espérer de la dissipation des malentendus? (1980: 210)

En d'autres termes, si les motivations, les désirs réels et les arrière-pensées des autres nous apparaissaient clairement, peut-être cesserions-nous de brasser des affaires et de croire en I'amour; le désespoir serait le prix de notre lucidité.

La plupart des travaux récents sur le malentendu postulent $q^{\prime}$ 'il s'agit non pas d'un accident de la communication, mais bien $d^{\prime}$ un phénomène inhérent au dialogue entre les humains ${ }^{1}$. Toutefois, le rejet d'un modèle idéal de communication ne conduit pas les théoriciens à déclarer qu'on n'y peut rien et qu'il en va mieux ainsi de toute façon. Le malentendu continue d'être perçu comme un phénomène fâcheux, comme un facteur à la fois d'incompréhension et de conflit. Sans écarter la possibilité $d^{\prime}$ un usage ludique et subversif du malentendu, surtout de la part d'artistes et d'écrivains, il reste que quelque chose pousse l'être humain à dissiper les malentendus dès qu'il en a conscience. Si le malentendu fait partie intégrante de la communication, il est aussi vrai de prétendre que les échanges verbaux tirent une grande part de leur dynamisme du souci d'en réduire le plus possible les effets pernicieux. On pourrait même postuler que ce qu'on appelle communément la pensée a beaucoup à voir avec la volonté de se libérer des leurres, des illusions et des malentendus qui bloquent notre accès à la fois au réel, à l'autre et à... nous-mêmes. 
Se pourrait-il en effet que l'on ne comprenne pas l'autre en premier lieu parce que l'on ne connaît pas son propre désir et sa place dans le monde? Combien de malentendus peuvent se glisser entre les amants, par exemple, lorsqu'ils se déclarent mutuellement leur amour? La phrase «Je t'aime» recèle trois zones de désaccords latents: qui est «je»? qui est «toi »? de quoi parle-t-on au juste lorsqu'il est question $\mathrm{d}^{\prime}$ " amour »? La littérature a de tout temps examiné des malentendus de cet ordre. Ils ne sont pas de ceux qu'une simple explication «métadiscursive » peut régler. Leur révélation nécessite du temps, le temps souvent que la rupture s'installe et qu'il devienne impossible de se parler en toute confiance (seule condition pour qu'un éclaircissement soit possible). Le malentendu révèle à la fois la solitude et la finitude du sujet: il doit en passer par le langage pour se faire entendre et cet accès au langage, jamais total, se heurte au langage tout aussi lacunaire de l'autre.

II n'est pas facile de départager, dans le malentendu, ce qui relève de la signification de ce qui aurait trait au désir ou au sentiment d'exister. Le «sens de la vie» n'est pas une question de sémantique! Jürgen Habermas postule, à la base de la communication, une "prétention à la validité »: on ne parlerait pas, on n'argumenterait pas si l'on ne voulait faire admettre quelque chose. Tout dialogue suppose donc la volonté d'établir un consensus. Cette approche rationaliste occulte un autre type de prétention que l'on pourrait nommer «prétention à la jouissance » : on parle pour éprouver le sentiment d'exister, pour jouir d'exercer un effet sur l'autre. Le sentiment de «ne pas être compris» renvoie non seulement au discours ou au message, mais aussi à la personne: ce qui est frustré, c'est le désir de reconnaissance du sujet parlant. C'est donc à travers le dialogue, au risque de tous les malentendus possibles, que le sujet élabore son identité et trouve sa place parmi les humains. Mais être pris pour autre que ce que l'on est, est-ce bien du malentendu? Ne s'agirait-il pas plutôt de méprise? Pour être exact, oui, mais nous verrons plus loin qu'une solidarité peut s'établir entre la méprise et le malentendu, au sens où notre réceptivité au discours de l'autre est en grande partie déterminée par l'idée que l'on se fait de sa personne.

Tous les malentendus ne sont pas tragiques et sans doute est-il assez fréquent que nous les jugions inoffensifs. Les malentendus ne deviennent un problème que s'ils menacent ou notre intégrité (réputation, dignité, etc.), ou les conditions de notre jouissance, de notre rapport au sens. C'est donc le malentendu en tant que source ou carburant du conflit qui retiendra mon attention. Dans cette perspective, il sera utile d'exercer notre regard critique et d'observer les lieux où le malentendu créateur de mésentente est susceptible de se glisser. II faudra évaluer en outre dans quelle mesure un effort de rationalisation permet d'élucider des modèles conflictuels déterminés à la base par du malentendu. D'entrée de jeu, il ne faut pas écarter la possibilité que certains de ces lieux, par delà ce qui s'annonce comme du malentendu (le fait de ne pas se comprendre, de se méprendre sur l'autre et sur son discours), conduisent sur les berges d'un autre genre de problème, celui d'une réelle incompatibilité entre discours qui rendrait impossible l'élection d'une règle rétablissant l'entente. Mais, dans la situation qui est la nôtre - celle, par exemple, où l'on voit de plus en plus s'établir un dialogue de sourds entre le monde judaïque-chrétien et le monde de l'Islam -, il paraît essentiel de réfléchir aux conditions de possibilité d'un dialogue qui assurerait la reconnaissance réciproque d'au moins quelques règles fondamentales.

Le langage que j'utilise dans cette étude n'est pas celui de la philosophie, mais j'aimerais souligner que les préoccupations philosophiques n'en sont pas entièrement absentes. Les enjeux théoriques les plus importants de ma réflexion vont à la rencontre, en effet, de la théorie de la communication énoncée par Jürgen Habermas (1991 et 1992 ; Cusset, 2001). Mon parti pris initial est d'accepter la théorie habermassienne comme on le fait d'une pétition de principe, mais d'en débattre certains aspects en lui soumettant des défis. Je discuterai en particulier trois principes qui me semblent problématiques: la place donnée à la raison spéculative et l'efficacité d'un métadiscours explicatif dans l'élucidation des malentendus ou dans la résolution des conflits; la possibilité d'un dialogue authentique dans certaines situations de conflit; le consensus comme visée ultime du dialogue.

Je retiens toutefois comme un apport essentiel la place que cette théorie accorde à la notion de règle. Il parait évident que, sans règle, l'humain ne pourrait fonctionner en société. Les règles et les normes d'action sont à l'humain ce que l'instinct est à l'animal: non seulement canalisent-elles ses pulsions et assurent-elles un minimum de cohésion dans le déroulement de ses activités, mais c'est par elles également que l'humain définit son identité, qu'il prend forme en tant que sujet. Un premier problème avec les règles est qu'elles sont $d^{\prime}$ 'une grande diversité, comme si aucune ne s'imposait d'emblée et de façon incontestable. Les règles sont toutes discutables en principe, bien que quelques-unes, érigées en lois, ne le soient qu'à certaines conditions. Un deuxième problème avec les règles est qu'elles doivent se fonder sur une légitimité que seule l'autorité, voire 
la force, peut lui garantir. Enfin, la multiplicité des règles et de leurs lieux d'application occasionne parfois des courts-circuits, lorsqu'une règle légitime entre en contradiction avec une autre tout aussi légitime, ou encore lorsque se croisent des genres de discours répondant à des règles hétérogènes.

Pour résumer, nous nous trouvons donc devant la situation suivante: 1) nécessité de règles dans tous les secteurs de la vie humaine; 2 ) relativité et variabilité des règles qui entraînent leur « discutabilité ». À cela s'ajoute un troisième aspect qui achève de compliquer les choses: la discussion sur les règles doit ellemême obéir à des règles. C'est précisément sur ce terrain que se situe la réflexion d'Habermas. Sans ignorer que, dans bien des cas, les règles sont imposées par la force, Habermas cherche à définir les voies d'un établissement des règles qui respecterait les principes de la démocratie et de l'égalité des sujets. Or, le seul terrain disponible pour la réalisation d'un tel projet est celui de la communication linguistique, de l'échange verbal. Respectant I'optique ouverte par Habermas et ses épigones, mon apport à cet effort de théorisation est celui d'un spécialiste en analyse du discours œuvrant depuis plusieurs années sur les modèles de communication conflictuels.

\section{La configuration du dialogue}

Penser le malentendu implique de penser plus généralement la situation dialogique, le rapport à l'autre dans I'acte de parole. Or, comme je le soutenais précédemment, ce rapport à l'autre est lourdement déterminé par le rapport que chaque sujet entretient avec lui-même. François Flahault (1978) a résumé la situation en montrant, dans sa théorisation des rapports de places, que trois questions fondamentales étaient en jeu dans toute relation: qui suis-je pour moi-même? qui est l'autre pour moi? qui suis-je pour l'autre? À partir de là, il devient possible d'interpréter le malentendu comme un écart entre représentations: I'autre ne me voit pas nécessairement tel que je m'imagine moi-même et vice versa. Mais si de tels présupposés sont susceptibles de produire des effets dans la réception du discours de l'autre, il ne faudrait pas pour autant limiter le dialogue à un jeu de miroirs. Le propre du langage, en effet, est de permettre de constants réajustements au sein des processus d'interprétation: si je m'aperçois que I'autre se fait des idées fausses à mon sujet, il est potentiellement loisible, par un acte de langage, de l'amener à modifier sa perception initiale. Une grande part de nos conversations a d'ailleurs pour fonction d'opérer ces réajustements. En revanche, il convient d'établir, comme base théorique de notre investigation, le caractère bipolaire du dialogue: le dialogue avec l'autre est doublé d'un dialogue interne avec soi-même. Qu'est-ce à dire, sinon que le "soi-même» en question est aussi un lieu de dialogue et le résultat de dialogues antérieurs: il y a de l'autre dans le «soi-même», et c'est parfois cet autre-là qui entre en conflit avec l'altérité que propose l'interlocuteur (voir Ricœur, 1990). Je ne suis jamais «in-divis» lorsque je dialogue avec l'autre, car ce que j'appelle «moi » est un lieu d'identifications et d'alliances diverses. En contrepartie, I'autre peut facilement devenir le réceptacle de projections, comme l'a depuis longtemps démontré la psychanalyse. En définitive, le dialogue met en jeu une activité de traduction et de négociation : je traduis le discours de l'autre à l'aide de catégories qui me sont familières, avec lesquelles je négocie également l'absorption de données qui m'étaient jusque-là étrangères. Ces processus peuvent être explicités par un acte de langage, mais, la plupart du temps, ils s'effectuent «en sourdine», chaque énonciateur étant persuadé de percevoir directement son vis-à-vis, sans aucune référence à des déterminations qui, d'ordinaire, lui échappent. Remonter jusqu'aux présupposés de nos jugements est un travail certes théoriquement possible, mais, dans la pratique, assez rarement réalisable. En effet, chaque énoncé d'un dialogue, chaque proposition, contient tant de possibles qu'il est impensable de suivre le fil de chacun.

Un autre facteur est à considérer: la présence d'un Tiers, notamment dans les situations dialogiques publiques. Parfois, le Tiers aura la fonction de médiateur ou d'arbitre, en autant que les co-énonciateurs le reconnaissent comme gardien d'une règle communément partagée. À d'autres moments, le Tiers sera plutôt un témoin. Sa seule présence, soulignons-le, peut entraîner des distorsions dans le discours dans la mesure où l'énonciateur, tout en s'adressant explicitement à un interlocuteur, s'adresse simultanément au Tiers. Or, la multiplication des destinataires (présents ou virtuels) est une source fréquente de malentendus. Si l'on peut en effet s'assurer d'être bien compris d'un interlocuteur unique, il devient pratiquement impossible de le faire avec des destinataires divers qui interpréteront le discours d'autrui à partir de catégories, de conventions et de présupposés hétérogènes.

Les dialogues publics à caractère idéologique présentent une autre particularité sur laquelle il convient de s'arrêter. La prise de parole, dans ces contextes, se déploie sur deux scènes ou deux espace-temps distincts: la scène interlocutoire ou pragmatique, d'une part, la scène discursive ou sémio-narrative, d'autre part. Cette position épistémique, défendue par certains linguistes et par la psychanalyse, concerne la stature même du 
sujet, que l'on considérera comme divisé, les deux scènes ne coïncidant jamais complètement. Linguistiquement parlant, il s'agit de faire la différence entre le sujet de l'énonciation et le sujet de l'énoncé. Dans l'argumentation, le sujet est à la fois un locuteur s'adressant à une ou plusieurs personnes et le sujet $d^{\prime}$ un discours qui déborde le plus souvent la situation déictique (ou interlocutoire). D'un point de vue rhétorique, la scène interlocutoire est le lieu où sont mis en jeu l'ethos et le pathos, alors que la scène discursive est celle du logos. Si le discours cherche à faire valoir une cohérence et vise une représentation totale du réel, l'interlocution, elle, est le lieu où se négocie la reconnaissance réciproque des interlocuteurs. Cette distinction est plus qu'une vue de l'esprit et permet d'identifier certains types de malentendus. II n'est pas rare que, croyant débattre d'idées et de "visions du monde», des interlocuteurs négocient en réalité leur place dans le monde en tant que sujets de parole. Bourdieu (1982) a bien montré que la portée d'un discours dépend en premier lieu de la crédibilité, de l'autorité et de la légitimité de celui qui le tient. Il a aussi soutenu que les prises de position étaient en partie ou totalement déterminées par la position sociale que l'on occupe. Toutes les prises de parole, en effet, ne sont pas égales de droit et les fameuses questions «D'où parlons-nous? » et "Qui sommes-nous pour parler?» interfèrent constamment sur le «Que disons-nous? ».

À partir de là, il nous faut insister sur l'ambiguïté créée par les deux sujets en présence: le sujet individuel qui prend la parole dans un cadre déictique bien précis et le sujet du discours que propose cette prise de parole. Le premier affirme sa prétention à la jouissance alors que le deuxième prétend à une validité qui se projette dans un autre espace-temps, à visée universelle et transhistorique. Prenons le cas du sujet d'énonciation. On peut espérer de ce sujet qu'il soit conscient, doué de raison et capable d'exposer toutes ses motivations; mais est-il théoriquement défendable de postuler ces attributs du sujet comme une condition initiale au dialogue authentique comme le fait Habermas? Tout au plus peut-on exiger du sujet qu'il tende vers cet horizon véridictoire. Le dialogue, nous en conviendrons, n'est pas un échange ou un débat autour d'idées toujours claires à l'esprit des locuteurs. II arrive qu'on s'adresse à l'autre pour cheminer vers sa propre parole, sans savoir exactement ce que I'on pense, mais en espérant que le dialogue permettra d'accéder à ce dire-vrai désiré. Ce processus est soumis à toutes sortes d'aléas et peut connaître des ratés: le sujet chemine, mais ses «erreurs de parcours » (gaffes ou maladresses d'expression) forment une mémoire malgré les diverses tentatives de réparation.
De par les interventions de l'interlocuteur, la sujet et sa parole subissent également des altérations, sont déstabilisés et doivent constamment se redéfinir.

Dans les conversations de la vie quotidienne, I'identification entre le sujet de l'énonciation et le sujet de l'énoncé est un fait linguistiquement attesté (on peut faire de soi l'objet de son discours), mais c'est tout de même dans les formes d'énonciation statiques (texte ou allocution) que le sujet de discours peut le mieux se constituer comme totalité cohérente (et, dans ce cas, la réplique intervient toujours en différé, ce qui occasionne d'autres types de malentendus). Ce sujet du discours n'est pas assimilable au sujet de l'énonciation; il s'agit au contraire d'une élaboration sémio-narrative, d'une forme de sublimation si l'on préfère. Par exemple, les traditions philosophique et scientifique ont longtemps cherché à mettre de l'avant un Sujet délivré des contingences individuelles et situationnelles: le sujet de la Vérité ou le sujet de la Science, sujets réputés universels et transhistoriques. À d'autres moments, des sujets d'énonciation prétendent parler au nom de groupes qui les incluent, voire d'instances symboliques complexes: «Telle est la ligne du Parti », «L'Église a parlé». Dans les poèmes engagés de Ronsard, par I'artifice de la prosopopée, la « France » devient sujet de discours. Enfin, on sait que, dans la tradition catholique, certains dogmes prétendent transmettre la voix de l'Esprit saint. Pour résumer, nous retiendrons comme pertinents quatre types de systèmes pouvant occuper la position de Sujet: I'individu, les communautés privées (couple, famille), les communautés fonctionnelles (entreprise, institution, corps de spécialistes), les communautés idéologiques (factions politiques, nations, religions, etc.). Dans I'univers de discours qui est celui de notre temps, ces différents systèmes sont à la fois autonomes et interreliés. Des tensions peuvent apparaître:

1) à l'intérieur de chacun d'eux (dans une entreprise, par exemple);

2) entre deux systèmes d'une catégorie de systèmes (par exemple entre deux entreprises concurrentes), ce qui nous oblige à penser qu'il existerait un système de systèmes (en l'occurrence le système régenté par l'économie de marché);

3) entre types de systèmes différents (ce que serait la tension, par exemple, entre les impératifs d'une entreprise et ceux de la famille, ou encore de la nation).

Dans le cas de relations qui s'étalent dans le temps, interfère également I'histoire de la relation. Chaque énoncé est jaugé à partir non seulement de son contenu sémantique, mais 
aussi du lien qui existe entre les co-énonciateurs. Sur ce plan intervient la question de la confiance réciproque, elle-même résultat de I'histoire relationnelle. À chaque occasion, il s'agit de mesurer les propositions discursives à l'aune des intentions que l'on prête à l'autre. Les malentendus associés à des «procès d'intention» nécessitent la plupart du temps une résolution non pas rationnelle, mais pragmatique. Supposons que j'aie reproché à quelqu'un la malveillance sous-jacente à l'une de ses interventions et qu'il proteste de sa bonne foi ; aucun argument rationnel ne me permet de démontrer la malveillance de son intention puisque celle-ci a été décodée à partir non pas des mots mais d'un « ressenti " provoqué soit par les intonations de son discours, soit par des présupposés que j'ai reconstruits par inférence. Ainsi, devant le déni de mon interlocuteur, je me vois contraint de décider s'il faut le croire ou non. Peut-être était-il réellement malveillant, le sait-il, mais décide-t-il de dénier la chose pour sauver la face, se promettant toutefois de faire attention à l'avenir. De mon côté, je peux aussi être conscient de ce jeu et «laisser passer » pour ne pas appesantir la relation. À ce stade, nous en sommes à déterminer non plus la vérité des faits (était-il malveillant ou non), mais bien le désir mutuel de sauvegarder la relation. Plus encore que la bonne foi, ce désir est à la base de toute réussite communicationnelle. Mais de tels incidents laissent des traces. De mon côté, par exemple, je retiens I'impression qu'une certaine méchanceté est présente chez mon ami et qu'elle pourrait se manifester à nouveau. De son côté, il peut se sentir vexé que je l'aie soupçonné de quelque chose et en conserver une pointe d'amertume, laquelle, éventuellement, alimentera un prochain facteur de ressentiment.

\section{LES TYPES DE MALENTENDUS}

Pour introduire à la complexité du malentendu, voici un court texte de Bernard Werber:

$$
\begin{gathered}
\text { Entre } \\
\text { ce que je pense } \\
\text { ce que je veux dire } \\
\text { ce que je crois dire } \\
\text { ce que je dis } \\
\text { ce que vous avez envie d'entendre } \\
\text { ce que vous croyez entendre } \\
\text { ce que vous entendez } \\
\text { ce que vous avez envie de comprendre } \\
\text { ce que vous croyez comprendre } \\
\text { ce que vous comprenez } \\
\text { il y a dix possibilités qu'on ait des difficultés à communiquer. } \\
\text { Mais essayons quand même... (2000: 34) }
\end{gathered}
$$

Cette maxime synthétise admirablement, à travers ses verbes, les principaux lieux du malentendu: le penser, le dire, le vouloir, I'imaginer, le croire, l'entendre, le comprendre. De l'acoustique au cognitif, en passant par des qualités qui touchent la personne même des interlocuteurs (leur volonté, leurs croyances, leurs désirs), on a là l'amorce d'une théorisation pertinente. La sagesse de cet axiome réside dans la prise en considération d'un écart, non seulement entre un "dire» et un "entendre » (axe interlocutoire), mais également entre le "dire» et le "vouloir-dire», phénomène qui s'inscrit cette fois au cœur même du sujet et consomme cette rupture entre la parole et le discours, entre l'énonciation et l'énoncé. C'est ainsi que le malentendu, parfois, est d'abord un «mal vu, mal dit», pour reprendre le titre d'un roman de Beckett. Mais il n'y a pas que le mal dit, il y a aussi du non-dit créateur de malentendus.

\section{Les malentendus pragmatiques}

Situation courante: croyant discuter d'opinions ou d'idées, des individus engagés dans un échange verbal se trouvent confrontés à un malaise indéfinissable qui bloque toutes les issues. La plupart du temps, le problème en est un de relation, de reconnaissance réciproque. Ce phénomène, pourtant si frappant, est bien souvent négligé par les spécialistes de l'argumentation et par les philosophes de la communication. C'est comme si un certain préjugé intellectualiste les portait à ne traiter le malentendu que sous l'angle du logique et du cognitif. Je soutiendrai contre eux que le ressort principal (et sans doute le plus prégnant) du malentendu doit être abordé du point de vue de I'interlocution, de ce que François Flahault appelle les rapports de places.

Ces rapports répondent à des préconstruits à la fois symboliques et imaginaires qui fixent, d'entrée de jeu, les modèles de représentations que les interlocuteurs entretiennent les uns par rapport aux autres. Ainsi, un ensemble de pouvoirs, de devoirs et de savoirs sont conférés à l'autre sur la base de sa position sociale: tel type de comportement est attendu, par exemple, de la part d'un médecin ou d'un professeur d'université, qui différera de celui que l'on attend d'un journalier. Cela touche non seulement la compétence, mais également le style, la manière de s'exprimer, les habitus (Bourdieu). Tout écart à ces modèles de représentation peut entraîner du malentendu.

À ces rapports symboliques sont associés des rapports plus étroitement imaginaires, au sens où ils sont déterminés moins par la fonction sociale que par une perception directe de l'autre (et de soi par rapport à l'autre). Ce type de perception met en jeu le 
désir (de soi et de l'autre). C'est ainsi que l'on est porté à juger l'autre inférieur ou supérieur à soi, compétent ou incompétent, inclus ou exclu du groupe auquel on prétend appartenir, etc. Il peut arriver à un arabophone d'avoir à expliquer, devant des interlocuteurs soupçonneux, qu'il n'est pas musulman, encore moins islamiste. Lorsqu'un politicien s'exprime sur la place publique (ou, comme cela arrive de plus en plus souvent, dans un talk-show), le fait-il toujours en tant que représentant d'un parti? Lui est-il loisible d'émettre une opinion individuelle ou doit-il s'en tenir à la « ligne du parti » ? Théoriser le malentendu, c'est bien sûr montrer comment se crée l'écart entre l'intention signifiante d'un énoncé et la manière dont il est interprété, mais c'est aussi, assurément, réfléchir sur la manière dont se définissent les rapports de place et les règles interrelationnelles - en d'autres mots, sur la manière dont se pose la question: que me veut l'autre?

La question très délicate de l'intentionnalité se joue sur cet axe relationnel. Comment savoir, en effet, si l'interlocuteur est de bonne ou de mauvaise foi, s'il obéit à des stratégies de manipulation, de domination ou de coopération? Ces questions sont étroitement reliées à la place que les interlocuteurs s'accordent réciproquement et au degré de confiance qui en découle. Les malentendus sur l'intentionnalité prennent prétexte de divers signes qui sont souvent paralinguistiques: inflexion de voix ou regard qui traduit un affect, silence suspect, geste révélateur, sourire, grimace imperceptible, rougeur, etc. Du côté du discours: parjure, écart entre le dire et le faire, équilibre instable entre le principe de franchise et le respect de la dignité de l'autre, etc. Dans la courte pièce de Nathalie Sarraute intitulée Pour un oui ou pour un non (1999)² , l'événement déclencheur de la remise en question du lien d'amitié entre H.1 et H.2 est une phrase banale, "C'est bien, ça », prononcée par $\mathrm{H} .1$ sur un ton que H.2 perçoit comme offensant. Le processus $d^{\prime}$ interprétation ouvert par cette phrase va si loin que les deux protagonistes sont conduits à conclure que l'histoire de leur amitié repose sur un malentendu initial jamais reconnu : aucun des deux n'accepte la place que l'autre tend à lui désigner. On peut se demander si de tels malentendus liés au désir, à la reconnaissance réciproque et au sens de la dignité peuvent être dénoués par un traitement autre que thérapeutique. La pièce de Sarraute est organisée pour montrer l'extrême difficulté d'y arriver de manière rationnelle et met en relief un phénomène que ne semble pas envisager Habermas: dans la communication, l'échange au sujet des problèmes relationnels n'interrompt pas l'interaction, ce qui veut dire en clair que les tentatives d'éclaircissement et d'élucidation des motivations, fussent-elles menées avec la meilleure volonté du monde, risquent, à l'insu des protagonistes, d'être minées elles aussi par la structure conflictuelle de base. La pièce de Sarraute permet de comprendre également que l'exigence d'un consensus peut parfois être, paradoxalement, un obstacle à la bonne entente. Savoir se reconnaître comme «non-ami » (sinon « ennemi ») est parfois un geste plus éthique que de lutter pour l'imposition $\mathrm{d}^{\prime}$ une règle commune.

J'appelle règles déontologiques les règles implicites ou explicites qui sont revendiquées sur l'axe de l'interlocution. Elles recoupent ce que Grice nomme des «maximes conversationnelles » dérivées d'un «principe de coopération » implicitement exigé des interlocuteurs. Selon Habermas, certaines attentes et présomptions sont inhérentes à l'acte de discuter, comme l'exigence d'être sincère ou de bonne foi, de reconnaître l'autre comme son égal, mais surtout de prétendre pour nos jugements à une validité universalisable. Face aux règles déontologiques, une démarche habermassienne consisterait en I'explicitation, si besoin est, de l'implicite qui entoure ces règles de manière à $s^{\prime}$ entendre sur leur respect. De fait, bien que ces règles agissent sur le plan de l'interlocution, elles peuvent néanmoins se retrouver sur le plan du discours lorsqu'elles sont mises en discussion, par exemple dans le cas où une règle aurait été enfreinte. Les partenaires du dialogue ont la possibilité de recréer un consensus autour de ces règles, peut-être même de les transformer. II en va de même pour tous les enjeux de la discussion : qu'ils soient affectifs, passionnels ou cognitifs, ils peuvent recevoir, dès que nous prenons part à une discussion, un traitement discursif qui les expose à l'examen rationnel ${ }^{3}$.

Le traitement discursif du déontologique montre qu'entre les deux scènes de l'interlocution et du discours, il existe des passages. Toutefois, la confusion entre les deux est lourde de conséquences. Quelle est la règle à partir de laquelle ceux qui discutent des règles déontologiques à respecter conduisent leur discussion? Nous dirons plutôt qu'ils sont guidés par une volonté, celle de trouver solution à leur problème. Pour y parvenir, ils doivent absolument examiner l'accroc à la déontologie qui aurait été commis comme un tort passager, l'isoler, si l'on peut dire, à la manière $d^{\prime}$ un médecin qui isole une cellule malade pour l'empêcher de contaminer l'ensemble du corps. À partir du moment où le tort est interprété comme une caractéristique inhérente au discours de l'autre (malveillance, mauvaise foi, etc.), celui-ci se transforme en adversaire et le dialogue devient impossible. Malheureusement, tous les protagonistes d'un dia- 
logue n'ont pas cette faculté de discuter un aspect irritant dans I'attitude de l'autre tout en maintenant la confiance à l'égard de son «soi profond».

Pour en rester aux malentendus relatifs à l'interlocution ou à l'acte de discours, on peut mentionner ceux que Marc Angenot (2001) nomme précisément des «malentendus de style pragmatique», parmi lesquels il inclut ces «maniérismes de la pensée et de l'expression » qui entraînent une interprétation de l'intentionnalité du locuteur et de son attitude générale en tant que sujet de parole. Angenot donne divers exemples: une bienveillance ostensible qui serait ressentie par l'autre comme manipulation méprisante; un refus de la brutalité intellectuelle qui serait perçu comme mollesse et indécision; une volonté de jouer franc-jeu qui, au contraire, serait reçue comme agression ou tentative d'intimidation. Dans des contextes discursifs déterminés par les conflits idéologiques, ce type de réception de l'acte de parole tend à recevoir un traitement lui aussi idéologique, ce qui nous donne un autre type de confusion possible entre le discours et I'interlocution. Par exemple, I'utilisation d'un certain lexique par un politicien sera jugée par ses adversaires comme «vile démagogie» ou «populisme de mauvais aloi». Du côté du féminisme, on jugera telle rhétorique «typiquement phallocrate», en deçà même des idées exprimées par le discours. C'est ainsi que, à tout moment, dans les échanges verbaux entre individus, le discours est-il résumé à certains vocables qui en traduisent la portée. On dira d'une intervention qu'elle est paternaliste, condescendante, méchante, affectueuse, bienveillante ou malveillante, autoritaire, noble, généreuse, mesquine, etc. À mon avis, cette perception quasi instantanée que l'on peut avoir d'une intervention est la principale pierre d'achoppement des approches intellectualistes du discours. Car le discours est d'abord reçu, qu'on l'admette ou non, de manière affective, la dimension logique passant au second plan. $C^{\prime}$ est ainsi qu'une intervention très articulée et imparable sur le plan logique peut agoniser sous les coups de récepteurs qui la jugeront avant tout "prétentieuse», «élitiste», «terroriste» ou «inutilement compliquée». Nonobstant le contenu, toutes ces qualifications se rapportent à l'acte de discours et donc à des variables énonciatives qui traduisent un conflit d'attitude entre le locuteur et le récepteur, d'une part, entre ces deux instances et les règles du discours, d'autre part.

Catherine Kerbrat-Orecchioni (1986) fait dériver le malentendu de ce qu'elle appelle le «calcul interprétatif » ${ }^{4}$. Son approche, résolument linguistique et pragmatique, ne s'attarde pas aux problèmes de surdité idéologique ou cognitive tels qu'on peut les observer dans les combats idéologiques, mais se penche avant tout sur les dissymétries, dans la conversation courante, entre l'encodage et le décodage. Ces types de problèmes se situent à la frontière de l'interlocution (qui met en jeu l'intentionnalité, les rapports de place, le mensonge, la bonne ou mauvaise foi) et du discours (où se mettent en place des éléments de contenu, la logique argumentative, I'interprétation sémantique des énoncés). Kerbrat-Orecchioni commence par dégager deux perspectives, celle de l'encodeur et celle du décodeur. Du côté du décodeur, elle distingue le contresens sincère, le contresens de mauvaise foi et le pseudo-contresens à fonction ludique. Le premier est l'équivalent de la méprise, du quiproquo involontaire. Par exemple, deux collègues sortent d'un cocktail et l'un demande à l'autre s'il est motorisé; I'autre répond «Oui, merci »; or, la question visait non pas à offrir un transport mais à s'en faire offrir un. Dans le contresens de mauvaise foi, le récepteur feint de ne pas avoir compris ou interprète volontairement «de travers». En exemple, cette conversation entre un parent et son enfant:

P: Je t'ai demandé de nettoyer ta chambre, je t'ai même montré
les moutons sous ton lit.
E: Justement, j'ai tout ramassé sous mon lit.
P: Oui mais ta chambre est encore en désordre!
E: Il fallait le dire que tu voulais que je nettoie toute ma chambre!

La mauvaise foi, ici, pervertit la compréhension à l'aide d'un distinguo sémantique qui paraît logique, mais qui, en fait, néglige volontairement la logique pratique. Enfin, le pseudocontresens à fonction ludique consiste à feindre la mauvaise foi, mais de manière à ce que cette feinte soit perçue comme une plaisanterie:

- Ferme la fenêtre, il fait froid dehors.

- Ah! penses-tu qu'il va faire moins froid dehors si je ferme la fenêtre?

Analysant les dissymétries du point de vue de l'encodeur, Kerbrat-Orecchioni est amenée à définir quatre niveaux d'interprétation dont la conjonction assure la communication réussie:

0- le dire apparent, ou explicite

1- la prétention signifiante: ce que $L$ veut faire croire ou admettre qu'il a effectivement dit (ce qu'il est prêt à assumer comme contenu de son dire)

2- l'intention signifiante: ce que $L$ veut faire ou laisser entendre

3- la pensée réelle de L. (Ibid.: 331) 
La différence entre 1 et 2 se joue sur la présence en 2 d'un implicite. Par exemple, dans l'ironie, le locuteur assume entièrement son dire, les mots qu'il a choisis, mais il veut faire entendre autre chose que ce qu'ils disent explicitement, cet autre chose étant bien souvent le contraire. Ou encore, si je dis à un membre de la famille: "Tiens, il n'y a plus de lait. Dommage que je ne puisse sortir en acheter, je dois surveiller les plats », il est fort probable que, par-delà l'information que je lui donne, je l'incite implicitement à me rendre le service d'aller lui-même en acheter. La mauvaise foi tire aussi ses effets de cette dichotomie, mais il s'y ajoute un mouvement secondaire de dénégation: le locuteur émet un énoncé derrière lequel il veut faire entendre autre chose, mais d'une manière suffisamment ambiguë pour qu'il ait le loisir ensuite de la dénier en cas de mauvaise réception. Par exemple,

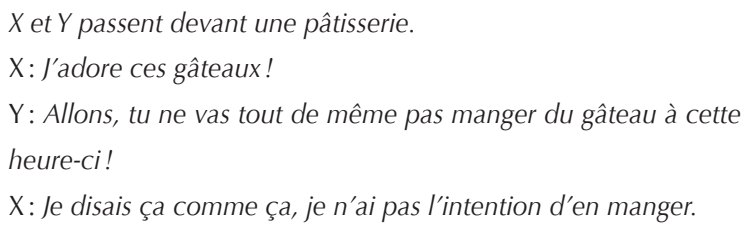

La rectification peut être de bonne foi, $\mathrm{X}$ ne faisant qu'informer I'autre de ses goûts. Si Y a correctement décodé l'intention signifiante, il ne réagit pas comme $X$ l'aurait souhaité, c'est-à-dire en donnant son accord au désir un peu coupable de $X$; cette réaction amène ce dernier à dénier la validité de l'interprétation de $\mathrm{Y}$. On voit donc que la mauvaise foi s'associe ici à un mensonge puisque $X$ cache sa pensée réelle. Le mensonge, en effet, présente un décalage entre 3 et 0-1-2. Tous ces exemples mettent en jeu l'implicite et c'est pourquoi la notion de «calcul interprétatif " s'avère pertinente: elle concerne autant les intentions du locuteur que la portée sémantique réelle de son discours.

Avant de passer aux malentendus fondés non pas sur une interprétation de l'intention, mais bien sur la signification des énoncés, quelques mots sur des cas où l'interprétation sémantique est déterminée par une perception partielle ou erronée d'éléments extra-discursifs. Du malentendu peut émerger par exemple d'une erreur sur le référent. Le roman de Benoît Duteurtre intitulé Les Malentendus présente un cas de confusion particulièrement ironique. Un Maghrébin nommé Rachid, qui vit clandestinement en banlieue de Paris, offre son bras à une vieille dame pour l'aider à traverser sans encombres un quartier dangereux. Elle le prend pour un «bon chrétien». Ils rencontrent un skinhead qui tourne vers eux « un regard suspicieux de défenseur de la race blanche», mais qui se retient d'agresser Rachid quand il s'aperçoit que la vieille dame est suspendue à son bras. Désignant le skinhead, la dame dit à Rachid : «Encore un Arabe! Méfie-toi, ce sont tous des voleurs. Ne leur fais jamais confiance...» (Duteurtre, 1999: 16-17). Autre exemple: dans une discussion qui possède son sujet, L2 enchaîne sur un sujet connexe, croyant que l'association d'idée sera perçue par L1, mais ce n'est pas le cas et L1 croit qu'il continue de parler du premier objet de la discussion. Ce type de méprise est en fait un cas de figure d'une classe plus générale, celle des malentendus occasionnés par une carence en information : comme lorsqu'on réagit aux propos d'une personnalité publique tels qu'ils ont été rapportés dans les médias, sans savoir qu'ils ont été incorrectement ou insuffisamment cités. Ce type de malentendu, de même que les deux précédents, peut facilement être éclairci à l'aide d'un métadiscours explicatif. Toutefois, il arrive que les situations $d^{\prime}$ interlocution ne permettent pas ce genre de rectification: un événement vient interrompre la conversation en laissant ouverte I'ambiguïté; la conversation elle-même dévie avant qu'on ait eu le temps de vérifier la validité des calculs interprétatifs; on refuse de donner crédit à celui qui se plaint d'avoir été mal cité. Ainsi, il peut se passer du temps avant que les choses ne s'éclaircissent, temps durant lequel le malentendu peut proliférer et entraîner des conséquences imprévues.

\section{Les malentendus sémantiques fondés sur des faits de langue}

J'insisterai peu sur les malentendus qui dérivent de problèmes acoustiques ou qui ont une origine strictement linguistique: « - J'ai tout fait pour lui. J'ai tout fait. - Si tu étouffais, tu n'avais qu'à t'en aller. » Ces malentendus, abondamment exploités par les humoristes, sont ceux qui ont davantage retenu les théoriciens du malentendu. Certains ont même fixé à trois le nombre de répliques nécessaires pour les corriger (Trognon, 2002; Weigand, 1999; Schegloff, 1992). Toutefois, il suffit de se pencher sur des exemples réels de malentendus (et non des exemples inventés par le linguiste) pour constater que, dans la plupart des cas, le sémantisme linguistique est doublé d'un sémantisme beaucoup plus difficile à cerner, d'ordre idéologique ou idiosyncrasique. Ce sont ces phénomènes qui retiendront mon attention.

Les mots sont dotés d'un potentiel polysémique qui oblige l'interprète à les comprendre « en contexte». La polysémie peut être inhérente à la définition même du mot. Par exemple, le mot « hôte» en français désigne autant celui qui donne I'hospitalité que celui qui la reçoit. La polysémie d'un mot peut être déter- 
minée par son histoire ou par l'usage qu'on en fait dans telle ou telle région. En général, les confusions engendrées par de telles variantes sont assez faciles à éclaircir, du moins lorsque les conditions dialogales le permettent. Récemment ${ }^{5}$, le metteur en scène et dramaturge québécois Robert Lepage déclarait que les habitants de la ville de Québec étaient «xénophobes », déclaration qui a suscité une levée de boucliers. Dans une lettre envoyée aux journaux, Lepage expliqua qu'il n'entendait pas dire que ses concitoyens étaient « racistes» car il avait utilisé le mot «xénophobe» en son sens étymologique: qui a peur des étrangers. II admettait qu'il s'agissait d'une erreur, car le mot désigne maintenant, dans I'acception courante, une hostilité et non simplement une peur.

D'autres effets de polysémies ou d'équivoques sémantiques dérivent $d^{\prime}$ un usage tropique du langage. Le trope, selon sa définition classique, consiste en une substitution: on dit une chose pour en signifier une autre, soit pour «faire image», soit pour éviter de nommer les choses trop clairement (pudeur ou respect humain), soit parce que la chose à exprimer échappe au langage commun. Dans la pièce de Sarraute, Pour un oui ou pour un non, H.2 veut expliquer qu'il s'est toujours tenu à l'écart de l'univers social et des valeurs de H.1, mais il a recours pour le dire à une métaphore: «Il faut vous dire d'abord que jamais, mais vraiment jamais je n'ai accepté d'aller chez lui... ». Ses interlocuteurs entendent la proposition au premier degré et lui demandent: "Vous n'allez jamais chez lui ? », ce qui oblige H.2 à préciser:

Ce n'est pas de ça que je parle. J'allais le voir. Le voir, c'est vrai. Mais jamais, jamais je ne cherchais à m'installer sur ses domaines... dans ces régions qu'il habite... Je ne joue pas le jeu, vous comprenez.

(Sarraute, 1999: 32)

On constatera ici que l' « explication » est davantage une amplification de la métaphore que sa traduction en termes dénotés.

Les problèmes de communication qui reposent sur des questions de langue, donc, peuvent être assez bien débrouillés par un acte métalangagier du type: «J'entendais ce mot non pas de cette manière mais plutôt dans le sens suivant... ». Toutefois, les choses se compliquent lorsque l'effort métalinguistique doit porter non plus seulement sur la dénotation d'un terme mais sur sa valeur discursive. À la confusion entre le littéral et le figuratif s'ajoute celle entre le péjoratif et le mélioratif implicitement associés à la connotation. Certains mots sont plus que des mots et font figure de notions ou de concepts; ce sont, en d'autres termes, des idéologèmes dont le sens déborde largement celui que leur donne le dictionnaire. Les mots ont une évolution: par exemple, les mots «bourgeois» et «fonctionnaire» ont glissé du statut de pures désignations sociologiques à celui de modèles de classification idéologiques. Angenot (2001) décrit aussi les polémiques au sein des mouvements socialistes autour de la notion $d^{\prime}$ « ouvrier». Les concepts de «totalitarisme» et de «fascisme» ont également subi une évolution du neutre au négatif. Mais cette évolution des concepts n'affecte pas que la connotation; elle peut aussi être le fait d'une redistribution des valeurs discursives dans I'espace social. Carl Schmitt (1992) fait remarquer à ce propos qu'une évolution - ni positive ni négative - a marqué le destin d'une notion telle que celle de "progrès ». Dans l'esprit des Lumières, le progrès était avant tout envisagé comme valeur morale référant au niveau de conscience de I'humanité. Au XIXe siècle, la notion a dérivé, sous la pression du libéralisme, vers des préoccupations économiques; puis elle a été adoptée par la science pour désigner l'évolution des moyens technologiques susceptibles d'améliorer les conditions d'existence. La notion de progrès fait partie de ces quelques vocables à vocation éminemment conflictogène parce qu'interprétables selon des perspectives très contrastées. Ce type de polysémie, on le voit, ne peut être réglé par le seul recours au dictionnaire et nécessite une approche du discours dans sa globalité. Nous devons, pour bien saisir le fonctionnement de ce dernier, considérer l'état du discours social, sa temporalité et sa spatialité. Les concepts, en effet, reçoivent des connotations différentes selon les discours qui forment l'univers discursif d'un espace social donné. À plus forte raison assiste-t-on à des disjonctions au sujet des concepts lorsque entrent en contact des discours issus d'espaces distincts (I'Occident et l'Orient, pour ne donner qu'un exemple extrême, mais ce pourrait être le cas entre l'Europe et l'Amérique, qui ne donnent pas la même valeur aux notions politiques de "gauche» et de «droite»). Pour illustrer un malentendu d'ordre culturel, Sybille de Pury donne I'exemple d'une femme africaine qui, s'adressant à des psychologues français, dit à propos de sa fille que «ça ne marche pas dans sa tête» (1998: 41). Pour les psychologues, cela signifie qu'elle est folle, alors que la mère veut faire comprendre qu'elle est sous l'emprise de la sorcellerie.

\section{Malentendus discursifs et coupures cognitives}

J'entends par «malentendus discursifs » les zones d'incompréhension qui mettent moindrement en jeu les faits déontologiques et concernent davantage le discours (ou récit constitutif) dans ses articulations sémantiques et sémio-narratives. 
1) Malentendus relevant de la mise en récit

La mise en récit des événements correspond à ce que les pragmaticiens de la communication (École de Palo Alto) nomment la «ponctuation des faits» (Watzlawick, Beavin et Jackson, 1967). Il s'agit pour le Sujet d'imposer une version des faits propre à lui assurer la position haute par rapport à ses opposants ou rivaux. Pour les belligérants, savoir qui est responsable du Tort initial fait toute la différence, car celui qui se livre à une agression doit la légitimer aux yeux des Tiers et le meilleur moyen de le faire est de démontrer que l'autre a commencé les hostilités. Parfois, la mise en récit doit comporter une dimension explicative et interprétative. C'est le cas lorsque le Tort initial n'apparaît pas à tous comme évident et doit donc être démontré. Par exemple, I'agression du 11 Septembre est perçue par la plupart des observateurs comme inaugurale, mais certains tentent de démontrer qu'elle n'est que la réponse à des agressions antérieures perpétrées impunément par les États-Unis. Toutefois, ce dernier point de vue nécessite une démonstration alors que le premier s'impose dans son évidence à quiconque ne possède pas une vue globale de l'histoire des relations entre les États-Unis et le monde arabe.

Mais il n'est pas nécessaire de recourir à des exemples si dramatiques pour montrer le fonctionnement de la ponctuation. À tout moment, dans nos échanges verbaux, nous effectuons des opérations qui provoquent des bifurcations dans le discours de notre interlocuteur. Ces mécanismes de sélection, de focalisation et d'occultation sont intrinsèques à la communication et ne sauraient être attribués à la seule mauvaise foi des locuteurs. À partir de quand nos répliques cessent-elles d'être pertinentes? Si le calcul interprétatif entre en jeu dans un simple énoncé, on peut imaginer alors la complexité créée par un enchaînement d'énoncés. Celui qui tente d'énoncer un point de vue organise son discours en fonction de la persuasion: il sélectionne les arguments et les exemples et il les présente dans un certain ordre; il crée entre eux des associations; il exploite les différents types de phrases disponibles comme l'interrogative, l'assertive, I'impérative, la constative; il parsème son discours de modalisateurs qui traduisent son degré d'adhésion à ses énoncés; il insiste sur certains aspects et passe rapidement sur d'autres. Son interlocuteur n'a pas le choix et doit, parmi cette abondance de signes possibles, effectuer une sélection et ce n'est pas toujours celle que souhaiterait le premier locuteur. Certaines assertions jugées centrales par celui-ci sont reléguées dans I'ombre (occultation) par celui-là, alors que des détails soi-disant insignifiants sont montés en épingle (focalisation).
Le degré de latitude de celui qui répond est très large. Tout en respectant la règle de pertinence, il peut à loisir faire dévier la conversation, entraîner l'autre sur des sentiers imprévus. On se demande pourquoi les débats publics tournent la plupart du temps en rond, et ce, malgré la présence d'un modérateur qui peut déclarer hors d'ordre certaines interventions. La raison principale réside en ces déplacements constants de la question, à ces recadrages qui redistribuent sans cesse la hiérarchie des arguments et des exemples.

Le malentendu peut s'immiscer dans l'échange lorsque, subrepticement, I'un des protagonistes opère un changement de niveau, en donnant par exemple à une assertion épistémique ( M. Untel commet une erreur logique en écrivant que...») une réponse déontologique (réponse de $M$. Untel: «On veut me faire passer pour un esprit embrouillé! »), ou en traduisant en termes idéologiques ( $X$ flirte avec la droite») une proposition axiologique ( $X$ avait simplement déclaré qu'il faudrait renforcer la conscience morale des citoyens). Font partie de cette catégorie les malentendus causés par la confusion entre le général et le particulier, la règle et le cas, l'acte et la personne. Ces malentendus renvoient pour la plupart à la logique interne de la langue et à la manière dont elle s'articule dans le discours. On comprend alors la passion des philosophes pour les systèmes qui réglementent l'enchaînement des propositions en établissant au préalable lesquelles sont jugées principales ou fondatrices, lesquelles sont secondaires ou dérivées. Cet effort de systématisation est en principe possible dans l'échange verbal, en autant bien sûr que les protagonistes fassent preuve d'esprit coopératif. Ce que je soutiens ici, c'est qu'il y a toujours du «reste», des questions latentes susceptibles de rejaillir à un moment ou un autre, du fait que les possibilités d'enchaînements, sans être infinies, dépassent largement ce que le sujet parlant, dans la finitude de son énonciation, est en mesure de récupérer. Ce fait, je m'empresse de l'ajouter, bien qu'il soit un facteur de malentendus (forcés d'aller de l'avant, nous ne pouvons nous permettre de tout rectifier et de légitimer tous nos présupposés), est aussi la condition de possibilité de l'échange, ce qui permet à l'un et à l'autre de trouver place dans le discours. Ce mouvement d'ouverture ou de dispersion établit une tension avec la visée totalisante des discours qui, au contraire, cherchent à contraindre au maximum la direction des enchaînements et à exercer ce que Fontanille appelle le "contrôle des isotopies" (1984). Dans cette perspective, avoir le dessus dans un débat n'équivaut pas nécessairement à produire les arguments les plus forts, mais avant tout à imposer ses propres isotopies, à régler 
soi-même le cadrage de la question. J'ajouterais : à imposer son style, son langage et les règles à partir desquelles sera jugée la validité des discours.

\section{2) Malentendus par confusion entre genres de discours}

Pour éviter les malentendus, n'est-il pas nécessaire de codifier rigoureusement et l'agir et le discours, de manière à ce que les critères de jugement soient clairs pour tout le monde? Savoir comment se comporter, quelle attitude adopter, voilà des inquiétudes typiquement humaines. Or, force est de constater que nous vivons dans un monde où les codes reliés aux pratiques et aux genres de discours sont bouleversés: une publicité télévisuelle, par exemple, met en scène les personnages d'un téléroman; ou encore, on voit dans un film de fiction apparaître le vrai Président des États-Unis.

Ces questions pourraient m'entraîner très loin ; aussi, je m'en tiendrai aux codifications entourant la pratique littéraire. L'un des combats de la modernité en littérature fut d'imposer au monde son autonomie par rapport à d'autres champs, particulièrement le politique et le religieux. Cette autonomisation était nécessaire à la fondation d'une institution spécifiquement littéraire dotée de règles autonomes. Les malentendus générés par ce passage furent puissants et, encore aujourd'hui, il est courant que des lecteurs interprètent des œuvres de fiction à partir de critères extra-esthétiques. On n'a qu'à penser au destin qu'ont connu les Versets sataniques et leur auteur, Salman Rushdie. Peut-on imaginer plus tragique malentendu 6 ? La contradiction va plus loin: au moins depuis les Romantiques, la littérature, tout en défendant âprement son autonomie, aspire également à agir sur le monde, à le transformer, voire à faire éclater sur le plan symbolique le principe de division qui fut pourtant nécessaire à son autonomisation. Au Québec, un écrivain comme Hubert Aquin rêve d'un texte révolutionnaire qui continuera de s'écrire «à la mitraillette», et il se désole d'une littérature rendue inoffensive par effet de cloisonnement dans un champ délimité au sein de la vie sociale. Bref, la littérature veut être reçue comme littérature, mais refuse qu'on dise d'elle qu'elle «n'est que littérature».

\section{3) Malentendus causés par l'imprécision des règles ou leur mise en contradiction}

Il faut évoquer aussi les malentendus qui tournent autour de la règle elle-même. Je relève deux cas possibles : celui où, sans s'en rendre compte, les protagonistes n'obéissent pas à la même règle de discussion; celui où le statut de la règle, sa définition, voire son existence, restent incertains. Pour illustrer le premier cas, prenons l'exemple d'une entreprise qui doit rencontrer les exigences du marché. Comme l'établissent deux chercheurs (Monroy et Fournier, 1997), un système doit satisfaire des impératifs divers : s'organiser et se structurer, échanger et produire, $\mathrm{s}^{\prime}$ inscrire dans le temps comme mémoire et comme projection. Les deux auteurs analysent une crise au sein d'une entreprise familiale fondée sur des principes de coopération et d'égalité. Dans son cadre initial, plutôt modeste, l'entreprise fonctionne bien, mais sa réussite l'oblige à une expansion qui compromet l'univers initial des valeurs. C'est ainsi que l'impératif de produire entre en conflit avec l'impératif de fidélité aux valeurs initiales: les contraintes du macro-système (le marché, fondé sur la compétition) obligent les sujets à abandonner une part de leurs idéaux. L'incertitude quant à la règle à privilégier (les deux règles concurrentes étant en principe légitimes) crée des motifs de malentendus qui peuvent dégénérer en conflit ouvert.

Plus grave serait le cas où se joue une indécision quant au fondement des règles elles-mêmes. J'ai mentionné plus haut ces cas où des arguments de nature cognitive, par exemple, sont interprétés à l'aide d'une grille de lecture axiologique. De manière plus radicale encore, Marc Angenot se demande si la raison, telle que définie par la tradition philosophique, est aussi universelle qu'elle le prétend $(2001 ; 2008)$. Sans nécessairement reprendre l'aphorisme de Pascal sur les « raisons du cœur», nous sommes appelés à nous demander, notamment à la lumière des relations entre l'Occident et l'Orient, si les modes de raisonnements admis par notre tradition peuvent seuls prétendre à la validité. Cela dit, même à l'intérieur de la tradition occidentale, I'insistance de la sophistique à miner la rationalité est là pour signifier la présence d'un Autre de la raison.

Sans prétendre résoudre la question de l'unicité ou de multiplicité des modes du raisonner, je pose quand même l'hypothèse de divergences déterminées en partie par la position des locuteurs les uns par rapport aux autres. Le défenseur d'un groupe dominé n'argumente pas de la même manière que le défenseur d'une idéologie au pouvoir. Celui dont la prise de parole n'est pas d'emblée rendue valide doit lutter pour se faire entendre, doit forcer la surdité volontaire des détenteurs du pouvoir et ceux-ci ont alors beau jeu de dénoncer son "agressivité » ou de l'écarter parce qu'il trouble «l'ordre public». Yves Cusset, pourtant émule d'Habermas, soulève la question de

[...] l'autoexclusion de ceux que leur condition ne peut conduire qu'à lutter contre la domination, à défaut de pouvoir jamais participer à la discussion. Jusqu'à quel point l'invitation au dialogue ne conduit-elle pas à dissimuler la réalité d'un mépris social ? Jusqu'où 
la sollicitation de la puissance autocorrectrice du langage peut-elle éviter le tort fait à ceux que leur refus de la toute-puissance du meilleur argument conduit au silence ou à l'exclusion?

(2001: 86)

Ces réflexions devraient nous permettre d'envisager la possibilité d'une éthique de la discussion qui ferait place à une posture agonistique. Voilà un projet sur lequel je reviendrai en conclusion.

4) Malentendus causés par la rigidité du cadre interprétatif

J'ai présenté plus haut l'affaire Rushdie comme un malentendu entre genres de discours, en l'occurrence le littéraire et le religieux. En note, je suggérais que la question était en fait plus complexe. Le problème est ici généré par la volonté, du côté du religieux, de s'inféoder tous les autres genres; l'impossibilité d'entendre l'opposant de la part du discours fondamentaliste provient donc de son intolérance à l'égard de toute forme de mise en question de ses dogmes et représentations. Pour ce genre de discours, donner le droit de parole à l'hétérodoxe, c'est comme introduire un poison dans un corps sain. À ce titre, même la réflexion que je mène maintenant, bien qu'elle ne prenne pas ouvertement parti pour l'un ou l'autre des discours, serait mal accueillie par un tel dogmatisme, du seul fait d'être un exercice de la raison, un exercice critique. Pour certains discours, il est des choses qui ne se discutent pas; pour eux, la Référence n'est pas la Raison mais la Parole de Dieu (ou du Prophète), ce que la Tradition a fidèlement transmis.

Or, l'essor des institutions guidées vers l'accomplissement de la raison dans la démocratie et le libéralisme a d'abord nécessité une séparation des champs de l'activité humaine. C'est ainsi que, peu à peu, la religion qui, comme son étymologie I'indique, servait depuis des temps anciens de fondement à la vie sociale, s'est vue reléguée dans la sphère privée. Elle ne devait plus interférer avec le politique et l'économique, le moins possible avec le culturel et l'esthétique. La division du social en champs ou sphères relativement autonomes entraîne une parcellarisation de la Référence qui occasionne des conflits entre règles hétéronomes. L'effort de la raison consiste donc à toujours repositionner les problèmes dans la sphère qui leur est propre (en évitant, par exemple, de traiter les choses politiques à partir de règles appartenant au religieux). Mais ce travail contient aussi sa part d'arbitraire et occasionne du conflit lorsqu'il s'agit de décider quelle règle (ou quel champ) aura prééminence sur les autres.
Il est loisible de repérer des schèmes rigides d'interprétation dans certaines pensées qui séparent de manière tranchée la droite et la gauche. Dans le roman de Benoît Duteurtre, le personnage de Martin obéit à un tel binarisme:

Selon le schéma politique de Martin, la "gauche» était le camp de la liberté (un monde sans frontières, une société fraternelle, la liberté sexuelle, la dépénalisation des drogues douces...); la droite celui du maintien de l'ordre (le travail, la famille, l'armée, la religion, la police...). (1999: 60)

Le roman présente un autre personnage, Camille, qui décode la réalité à partir du concept de « fascisme » dans un amalgame où se fondent une série de représentations hétérogènes:

Le même mot [fasciste] ponctuait sa conversation pour désigner non seulement l'extrême droite politique - les nostalgiques de Pétain ou de Mussolini qu'elle estimait innombrables - mais aussi les conservateurs bon teint, les progressistes modérés et toute personne qui lui déplaisait moralement et physiquement. Elle avait un jour dénoncé comme "fasciste » une employée de banque qui la mettait en garde contre des découverts trop fréquents. Pour Camille, ce comportement rejoignait l'indifférence des fonctionnaires nazis sous le III Reich. Ne pas voir le fascisme embusqué dans nos moindres actes, tel était, selon elle, le symptôme premier $d u$ fascisme. (Ibid.: 84-85)

Est-il simplement possible d'envisager la mise en discussion d'une telle logique qui incrimine à l'avance toute remise en question de ses présupposés?

\section{LE MALENTENDU COMME INDICE DE LA FINITUDE}

On ne peut servir toutes les valeurs à la fois.

(Ricœur, 1990: 302)

La section qui précède m'a permis d'établir à quel point la notion de «malentendu » pouvait agir sur de multiples plans. $S^{\prime}$ il est relativement facile de réparer des malentendus linguistiques (compréhension d'un mot dans son sens dénoté), nous avons vu que la réparation devient plus ardue, parfois même improbable, dans le cas des phrases complexes mettant en jeu des représentations, des croyances, des valeurs sémantiques informées par I'idéologie, etc. J'ai aussi cherché à montrer qu'à ce plan de la signification se juxtapose celui du désir et qu'une contamination de l'un par l'autre peut considérablement brouiller la communication. Lorsque cela se produit, les demandes d' « explication», de «justification», de «précision » demeurent hasardeuses étant 
donné que tous ces «métadiscours » continuent d'être produits par des sujets en relation intersubjective, animés par des désirs et des peurs, pressés par des intérêts économiques ou symboliques, orientés mentalement par des schèmes préconstruits qui déterminent leur aperception de la réalité. Ces phénomènes, loin d'être tributaires de pathologies, forment I'ordinaire de la communication. Selon Lyotard (1983), le potentiel de différend serait inscrit dans le fonctionnement de la langue elle-même, toujours lacunaire à l'égard du réel. Le réel échappe à la prise du sujet du fait qu'aucune prise de parole n'est capable d'exploiter à la fois tous les genres de discours, de réaliser toutes les valeurs et d'harmoniser toutes les antinomies (celles de la métaphysique classique: la quantité et la qualité, I'homogénéité et I'hétérogénéité, la rupture et la continuité, I'expansion et la concentration, etc.). Aucun discours ne peut prétendre, de par l'impératif qui est le sien de se construire une cohérence, occuper le champ entier des possibles sémantiques: toute cohérence implique le sacrifice d'une part du possible. À partir de là, ne peut-on pas postuler que le geste éthique consistera à poster le sujet devant cette impossibilité en créant une forme qui, plutôt que de gommer la finitude, mettrait en scène sa loi et le déchirement qu'elle provoque dans le discours? Ne doiton pas en arriver à la conclusion que, parallèlement au travail de la raison, une certaine posture agonistique peut se greffer à une éthique de la communication?

La posture agonistique, telle que je cherche à la définir ici, ne signifie en rien le rejet des règles au profit d'une authenticité qui se développerait en dehors d'elles. Elle consiste plutôt à déboulonner sans arrêt l'autoritarisme de la règle unique ou dominante. La littérature peut accomplir ce genre de tâche, en provoquant par exemple un renouvellement du regard posé sur les formes qui dictent des comportements. L'un des personnages du roman de Duteurtre exprime clairement ce que serait I'éthique du roman, ce geste de mise en suspens du jugement: «Tu devrais réfléchir aux bizarreries de notre époque, au lieu de répéter des formules toutes faites» (1999: 86). Dans la sclérose des discours prisonniers de leurs mécanismes, l'agôn introduit du jeu, et je voudrais qu'on entende ce mot dans au moins deux acceptions que lui donne le français, soit celle d'une activité ludique obéissant à des règles (toujours en cours de redéfinition), mais également celle d'un espace ou d'un relâchement créé entre deux objets qui sont fixés l'un à l'autre. "Avoir du jeu», dans cette dernière acception, c'est avoir l'espace nécessaire pour manœuvrer et c'est, par extension, pouvoir prendre ses distances à l'égard d'un phénomène de manière à s'en jouer, tout en le prenant au sérieux. Dans la mesure où l'acte critique ne peut prétendre avoir lieu en dehors de la situation conflictuelle qu'il veut dénouer, l'agonistique est cette posture qui, depuis le lieu du conflit, en déjoue le caractère mortifère en y introduisant du jeu. Il y a d'ailleurs dans l'étymologie du mot grec agôn une association entre les isotopies du combat, de l'angoisse reliée à la mort et du jeu réglé.

Du point de vue non seulement de la littérature mais également des délibérations entourant les modes de la socialité, je ne peux entériner entièrement les propositions habermassiennes car, de réduire l'agonistique aux volontés dominatrices et égocentriques des sujets, elles négligent la possibilité d'une éthique qui adopterait à l'occasion la voie du combat. Ce faisant, la théorie habermassienne soumet sa validité à des a priori que les situations réelles d'échange peuvent rarement rencontrer, ce qui la rend inefficace dans la plupart des discussions, qu'elles soient publiques ou privées. Si la posture agonistique ne donne pas le primat à la raison comme Référence ultime du jugement, elle ne la rejette pas pour autant; elle ne fait qu'en relativiser la portée à l'égard des tensions qui habitent l'être humain. Le mouvement argumentatif met inévitablement en jeu des critères de raison, mais là où la théorie habermassienne est piégée par un préjugé intellectualiste, c'est lorsqu'elle en arrive à réduire la discussion à l'argumentation et à la justification devant la Norme.

Je crois avoir montré que les arguments ne sont qu'un des lieux parmi d'autres où les sujets peuvent se rencontrer (ou non). Il faut se rendre compte qu'argumenter, parfois, ne donne strictement rien, voire envenime les situations. Théoriquement parlant, l'une des conclusions à tirer de toutes ces réflexions serait donc que, simultanément à ce que Habermas nomme prétention à la validité, la prise de parole est traversée tout aussi fondamentalement par une prétention à la jouissance, ce dernier terme incluant des visées aussi diverses (mais toutes solidaires) que l'aspiration à la dignité, la quête de reconnaissance et le désir de complétude. À la jonction de ces deux prétentions peuvent s'élaborer de nombreux et tenaces malentendus.

Aux exigences de la raison, il faut associer celle du désir car c'est sur ce plan que s'élaborent la plupart des malentendus. Les situations de malentendus créées par le désir atteint de cécité rendent inefficace le recours à la discussion fondée sur l'échange d'égal à égal d'arguments dûment justifiés, et ce, d'autant plus que les sujets sont engagés dans un conflit:

Lorsqu'un conflit se noue autour d'une injustice, chacune des deux parties tend à adopter une posture d'affrontement. [...] Lorsque nous sommes pris dans un conflit, nous risquons fort 
de méconnaitre la tension ou la division inhérente à notre désir - désir de coexistence et de justice, mais en même temps désir de supprimer l'autre, de l'écraser. Dans la conscience que nous avons de nous-mêmes, cette division tend à s'estomper car la frontière entre les deux désirs, au lieu de passer à l'intérieur de nous, nous paraît passer à l'extérieur de nous: à nos yeux, elle se confond avec la frontière qui nous sépare de l'autre partie. Ainsi, une fois entrée dans un scénario d'affrontement, chacune des deux parties en cause se voit dotée d'une entièreté (et par conséquent d'une jouissance de soi) que l'état de paix lui refuse.

(Flahault, 1998: 186)

Dans son analyse de talk-shows télévisés, Galatolo fait aussi remarquer que, dans les échanges orientés vers un accord, les interlocuteurs privilégient l'harmonie de la relation à la dénonciation des malentendus, ce qui est plus improbable dans les situations orientées vers le conflit. Ses observations la conduisent même à observer que, dans les échanges conflictuels, les participants utilisent la réparation des malentendus comme des ressources pour attaquer l'autre (2003: 75-77).

Je conclus cet article en suggérant que tout malentendu n'a pas à être élucidé, que ce qui échappe au contrôle de notre raison peut aussi devenir facteur de liberté, en autant que ce qui nous sépare de l'autre ne soit pas renforcé par le déni de la finitude (de notre langage, de notre être). Les effets pernicieux du malentendu se font davantage sentir lorsque les interlocuteurs méconnaissent sa présence et sont persuadés d'avoir décodé parfaitement le discours de l'autre. La seule évocation du malentendu, par un des protagonistes d'un conflit, trace la voie vers sa résolution, du moins vers une mise en suspens des éléments conflictuels. Si le conflit est inévitable, on peut espérer au moins qu'il ne soit pas déterminé par notre ignorance de ce que l'autre prétend signifier.

\section{NOTES}

1. C'est le cas avec Jankélévitch, comme nous venons de le voir, qui adopte une perspective psycho-esthético-philosophique (perspective qui sera systématisée par Lyotard dans Le Différend). Mais même les pragmaticiens, désireux de trouver les voies d'une résolution des malentendus, en conviennent (voir Weigand, Trognon, Schegloff, Galatolo et Dascal). Plus récemment, I'analyste du discours Marc Angenot (2008) a poussé le postulat jusqu'à son extrême limite en remettant en question le pouvoir qu'aurait la rhétorique argumentative de conduire au règlement des conflits et malentendus.

2. Pour une étude détaillée de cette pièce, en rapport avec la question du malentendu, voir Garand (2003).

3. Le fait de s'en remettre à une discussion guidée par la raison, comme je l'établirai plus loin, n'assure pas pour autant l'entente. Qui dit raison dit raisonnement, donc des règles du «bien raisonner»sur lesquelles tout le monde ne s'entend pas. Cette difficulté fait l'objet du dernier livre de Marc Angenot (2008).

4. Je lui emprunterai, dans les pages qui suivent, quelques exemples pertinents.

5. C'est-à-dire en octobre 2006.

6. Mais j'aimerais préciser que, dans ce cas, le mal entendre est doublé d'un bien entendre. Il y a malentendu dans le fait que Rushdie ne se moque pas lui-même en personne de Mahomet et qu'il faut, pour comprendre le passage litigieux de son livre, tenir compte des médiations qu'instaure la fiction: le personnage, le rêve, etc. Toutefois, les fondamentalistes ont raison de dire que la démarche même de Rushdie est hérétique par rapport à leur vision du monde, car cette dernière leur refuse même le droit de soumettre Mahomet à un traitement fantaisiste. Pour eux, la liberté que prend Rushdie face à cette figure, toute fictionnelle qu'elle soit, est inacceptable. À ce niveau, il ne s'agit plus de malentendu mais de coupure cognitive. 


\section{RÉFÉRENCES BIBLIOGRAPHIQUES}

ANGENOT, M. [2001] : «Dialogues de sourds : doxa et coupures cognitives", Discours social/Social Discourse (Cahiers de recherche), nouvelle série, vol. 2, Montréal;

[2008] : Dialogues de sourds. Traité de rhétorique antilogique, Paris, Mille et une nuits, coll. «Essai ».

AQuin, H. [1995] : Prochain Épisode, éd. critique établie par J. Allard, Montréal, Leméac, coll. "Bibliothèque Québécoise».

Baudelaire, C. [1968] : CEuvres complètes, Paris, Seuil, coll. «L'Intégrale».

BOURDIEU, P. [1982] : Ce que parler veut dire. L'économie des échanges linguistiques, Paris, Fayard.

CUSSET, Y. [2001]: Habermas: l'espoir de la discussion, Paris, Éd. Michalon.

DASCAL, M. [1999] : «Introduction: some questions about misunderstanding ", Journal of Pragmatics, vol. 31, no 6, 753-762.

DUteUrTRE, B. [1999] : Les Malentendus, Paris, Gallimard.

Flahault, F. [1978] : La Parole intermédiaire, préface de R. Barthes, Paris, Seuil;

[1998] : La Méchanceté, Paris, Descartes \& Cie.

FONTANILLE, J. [1984] : "Cause toujours... je focalise". Remarques sur la polémique conversationnelle», Actes sémiotiques, no 30, 44-53.

GALATOLO, R. [2003] : «Le malentendu en milieu conflictuel : une révision du cas standard», dans M. Laforest (dir.), Le Malentendu : dire, mésentendre, mésinterpréter, Québec, Nota bene, 65-93.

GARAND, D. [2003] : «Le théâtre du malentendu », dans E. Tarasti (dir.), Acta Semiotica Fennica XVI : Understanding/Misunderstanding, Contributions to the Study of the Hermeneutics of Signs, Helsinki, International Semiotics Institute at Imatra, 153-164.

GRICE, H. P. [1975] : «Logic and Conversation», dans P. Cole et J. L. Morgan (dir.), Syntax and Semantics, vol. 3, New York, Academic Press, 41-58.

HabeRMAS, J. [1991] : Morale et Communication, Paris, Éd. du Cerf;
[1992] : De l'éthique de la discussion, Paris, Éd. du Cerf. JANKÉLÉVITCH, V. [1980]: Le Je-ne-sais-quoi et le Presque-rien. 2. La Méconnaissance. Le Malentendu, Paris, Seuil, coll. «Points». Kerbrat-OreCCHIONI, C. [1986] : L'Implicite, Paris, A. Colin. LYOTARD, J.-F. [1983] : Le Différend, Paris, Minuit, coll. «Critique». Miguelez, R. [2001] : Les Règles de l'interaction. Essai en philosophie sociologique, Québec, Les Presses de I'Université Laval.

MONROY, M. et A. Fournier [1997] : Figures du conflit. Une analyse systémique des situations conflictuelles, Paris, PUF.

PURY, S. de [1998] : Traité du malentendu. Théorie et pratique de la médiation interculturelle en situation clinique, Le Plessis-Robinson, Institut Synthélabo pour le progrès de la connaissance, coll. «Les Empêcheurs de tourner en rond».

RICCEUR, P. [1990] : Soi-même comme un autre, Paris, Seuil.

RuSHDIE, S. [1989] : Les Versets sataniques, Paris, Christian Bourgois. Sarraute, N. [1999] : Pour un oui ou pour un non, Paris, Gallimard, coll. « Folio théâtre ».

SCHEGLOFF, E. A. [1992] : « Repair after the next turn : the last structurally provided defense of intersubjectivity in conversation », American Journal of Sociology, vol. 97, no 5, 1295-1345.

SCHMITT, C. [1992] : La Notion de politique. Théorie du partisan, Paris, Flammarion, coll. «Champs».

TROGNON, A. [2002] : "Speech acts and the logic of mutual understanding », dans D. Vanderveken et S. Kubo (dir.), Essays in Speech Acts Theory, Amsterdam, John Benjamins and Sons, 121-133.

Watzlawick, P., J.H. Beavin et D. D. Jackson [1967]: Pragmatics of Human Communication. A Study of Interactional Patterns, Pathologies, and Paradoxes, New York, W. W. Norton \& Company Inc.

WEIGAND, E. [1999] : "Misunderstanding: the standard case», Journal of Pragmatics, vol. 31, no 6, 763-785.

WERBER, B. [2000] : L'Encyclopédie du savoir relatif et absolu, Paris, Albin Michel. 